\title{
Promises and pitfalls of digital knowledge exchange resulting from the COVID-19 pandemic
}

\author{
Vivian M. Nguyen ${ }^{1,2}\left(\right.$ C Christina Bell ${ }^{3} \cdot$ Valerie Berseth $^{2} \cdot$ Christopher Cvitanovic $^{4} \cdot$ Ray Darwent $^{5}$. \\ Matthew Falconer ${ }^{6} \cdot$ Jenna Hutchen ${ }^{2} \cdot$ Tyreen Kapoor $^{2} \cdot$ Nicole Klenk $^{7} \cdot$ Nathan Young $^{3}$
}

Received: 25 July 2021 / Accepted: 11 October 2021 / Published online: 25 November 2021

(c) The Author(s), under exclusive licence to Springer Nature Singapore Pte Ltd. 2021

\begin{abstract}
In this article, we integrate our authorship experiences with insights from nine interviews of knowledge exchange practitioners at the Canadian Forest Service about challenges and opportunities of digital knowledge exchange (KE) brought on by the COVID-19 pandemic. We aim to inform how best to maintain effective KE practices and processes in a digital-first world. Interpersonal trust and relationships are pivotal to effective knowledge exchange; thus, removing these dimensions risks losing aspects of social learning, informal and meaningful discussions, and personal connections that affect how we interpret and respond to subtle affective and social cues. For KE practitioners, lack of in-person interactions risks internal KE coordination and relevance of KE work, and diminished ability to predict and respond to user needs. However, the accelerated digital adoption has increased reach and accessibility for diverse people to exchange knowledge, and enables more frequent and rapid response to issues and events by virtually gathering diverse people almost instantly. The acceleration in digital innovation and culture has thus resulted in new tools and diversified approaches for the KE toolbox to inform decisions and practices. The long-term sustainability and effectiveness of digital KE depend on two interconnected factors: addressing the persistence of the digital divide and people's abilities to make and maintain meaningful social connections in the absence of regular face-to-face contact. We thus offer three considerations to guide KE efforts and initiative in a digital-first world: (1) consider both digital divide and equity; (2) revisit user needs and preferences for KE to address the diversity of users, and (3) leverage the diversification of KE approaches and innovations.
\end{abstract}

Keywords Knowledge mobilization $\cdot$ COVID-19 $\cdot$ Digital communication $\cdot$ Science communication $\cdot$ Digital diversification $\cdot$ Engagement $\cdot$ Interpersonal trust $\cdot$ Science transfer $\cdot$ Environmental management $\cdot$ Forestry $\cdot$ Conservation

Vivian M. Nguyen

vivian.nguyen@carleton.ca

1 Institute of Environmental and Interdisciplinary Science, Carleton University, 1125 Colonel By Drive, Ottawa, ON K1S5B6, Canada

2 Biology Department, Carleton University, 1125 Colonel By Drive, Ottawa, ON K1S5B6, Canada

3 School of Sociological and Anthropological Studies, University of Ottawa, 120 University Private, Ottawa, Canada

4 Australian National Centre for the Public Awareness of Science, Australian National University, Canberra, Australia
5 Natural Resources Canada - Canadian Forest Service, Northern Forestry Centre, 5320-122nd Street, Edmonton, AB T6H 3S5, Canada

6 Natural Resources Canada - Canadian Forest Service, 580 Booth St, Ottawa, ON, Canada

7 Department of Physical and Environmental Sciences, University of Toronto Scarborough, 1065 Military Trail, Toronto, ON M1C 1A4, Canada 


\section{Introduction: knowledge exchange-an interpersonal activity}

It is widely accepted that efficient and effective knowledge exchange among scientists and decision-makers helps to improve outcomes in environmental management and conservation (Fazey et al. 2012; Cvitanovic et al. 2015; Nguyen et al. 2017). Knowledge exchange (KE) has many definitions and interpretations, but at its core, it implies a two- or multi-way reciprocal and iterative exchange of knowledge and learning (Fazey et al. 2012, p. 20; Nguyen et al. 2017, p. 791). Effective knowledge exchange helps people with different knowledge needs to learn from one another, thus building increased capacity for evidenceinformed policies and practices and enhancing the legitimacy of decision-making processes.

Theories of KE stress the importance of social connection and collaboration for individual and collective learning (Evely et al. 2012, p. 27; Fazey et al. 2012, p. 23; Cvitanovic et al. 2021, p. 183). While some learning is linear and codified (i.e. coming from documents), KE conceptualizes learning as dynamic and relational, coming from iterative and multidirectional interactions (Nguyen et al. 2017, p. 794). Successful KE gives people access to a range of viewpoints and different types of knowledge and interpretation beyond their own personal and professional experiences and networks (Ward et al. 2012, p. 302). The extent to which KE participants successfully communicate and exchange information is dependent on their ability to foster relationships founded on shared goals, mutual respect, and trust (Addison et al. 2013, p. 496; Reed et al. 2014, pp. 341-343; Cvitanovic and Hobday. 2018, p. 2).

As such, $\mathrm{KE}$ is most effective in the context of strong interpersonal relationships that are founded upon shared goals, mutual respect, and high levels of trust (Young et al. 2016; Cvitanovic and Hobday 2018, p. 4). Traditionally, such relationships have been forged by networking, collaborations, and efforts to coproduce knowledge-processes that rely heavily on in-person and interpersonal interactions (Nguyen et al. 2017, 2019, Cvitanovic et al. 2016, p. 869; Chambers et al. 2021, p. 5). For example, a recent investigation of one of the worlds oldest boundary organizations focused on improving KE at the interface of science and policy found that efforts to build trust between individuals and organizations is best achieved through a combination of formal and informal in-person, face-toface interactions, particularly among stakeholders that have differing worldviews (Cvitanovic et al. 2021, p. 184). This is not to say that-in the absence of in-person interactions such relationships cannot be formed-but rather that importantly, each of these social rituals of relationship building that are known to be most effective have been compromised by COVID-19 pandemic, which has forced a digital transformation in work and collaboration due to stay-at-home orders and travel restrictions (Schwarz et al. 2020). As a result, knowledge exchange research and practices must be re-examined, especially when digital KE is more common now than before. Even though KE has been shown to be most effective in-person, the acceleration of digital work spurred by the pandemic leads us to ask how has it affected KE and what can we see as new approaches to KE?

Our Perspective Essay examines how knowledge exchange principles may need to be re-examined through COVID lenses by discussing potential short- and long-term implications of this digital transformation of KE spurred by the COVID-19 pandemic. We stress that these implications can be both positive and negative for KE processes and outcomes. On the one hand, the absence of in-person interactions may result in fewer opportunities to build relationships and trust, present challenges for equity and equality, and limit the efficacy of knowledge communication and learning (Barnes 2020; Beaunoyer et al. 2020; Nguyen et al. 2020; Schwarz et al. 2020). On the other hand, the digital transformation of KE may present opportunities such as reduced barriers to participation, present opportunities to assemble and hear from broader and more diverse groups, and create opportunities to meet more frequently via video conferences. For example, Niner and Wassermann $(2021$, p. 7) found that moving marine-related conferences online increased their accessibility to those who would have been unable to attend for financial or personal reasons. However, while recent research such as this has begun to understand the advantages and disadvantages of digital KE, our understanding of these issues remains in its infancy, and are based only on a small number of case studies, thus suggesting that additional work is needed to understand these issues across different contexts.

To this end, the overarching aim of this Perspective Essay is to contribute to the growing literature about the digital transformation of KE. We do so by drawing on the experiences and expertise of the authors, all of whom are active in both the research and practice of KE. To enhance our perspectives, and ensure novel insights are added through this Perspective Essay, we also integrate a case study from the Canadian Forest Service (CFS). Specifically, we describe and discuss the outcomes of interviews with KE practitioners from the CFS on the impacts and challenges of digital KE as a result of the COVID-19 pandemic. Here, a KE practitioner is considered an individual who implements knowledge exchange strategies to bridge relevant knowledge between producers and users. By combining our perspectives with the case study, we are able to identify and discuss bright spots and opportunities for the digital transformation of KE. Specifically, we ask the following questions about the 
fate of KE in a digital-first world: what is gained and what is lost with digital KE? Is there an optimal way to leverage opportunities of digital interaction for improved KE access and participation? Is there a tipping point where digital $\mathrm{KE}$ becomes less effective or ineffective for some participants? How can remote communities with digital access challenges be engaged in digital KE?

By answering these questions, this perspective article aims to provide practical advice and guidance for how best to maintain effective KE practices and processes in a digitalfirst world. The article proceeds in the following manner: In Sect. 2, we describe the case study of the CFS and the data collected from KE participants in our research. In Sect. 3, we report on the perspectives of KE practitioners at the CFS on the challenges to KE caused by the COVID-19 pandemic. Section 4 draws primarily on authors' perspective using CFS examples to discuss the risks and opportunities that emerge from the ways that KE practices have changed during the pandemic. Finally, Sect. 5 identifies what we judge to be constructive changes and how those will impact future digital KE work, while Sect. 6 presents concluding thoughts on post-pandemic considerations for KE.

\section{The case: the Canadian Forest Service KE practitioners}

In Canada, forest management is the jurisdiction of provincial governments. However, forests themselves do not respect these jurisdictional boundaries, meaning there is a need for national-level coordination. As such, the federallevel agency, The Canadian Forest Service (CFS), ${ }^{1}$ plays a key role in coordinating national forest policy and management. Additionally, the CFS has a mandate to fund and conduct forestry research relevant to policy. Thus, while the CFS has no jurisdiction over forestry practices, it plays an important role in science, advice, and innovation (Hardy 2002 p. 57; Klenk and Hickey 2011).

An important CFS strategy for communicating and exchanging scientific knowledge is through designated individuals known as "knowledge exchange specialists" (herein referred to CFS KE practitioners). CFS KE practitioners work in six regional research centres across Canada in addition to their head office located in Canada's Capital, Ottawa. The role of the CFS KE practitioners is to act as a liaison between CFS scientists and anyone interested in using the knowledge they produce (e.g. industry, non-governmental organizations, provincial government, Indigenous communities, academia, other governmental departments). These KE

\footnotetext{
1 https://www.nrcan.gc.ca/our-natural-resources/forests-forestry/thecanadian-forest-service.
}

practitioners make the science accessible to a non-scientific audience by, for example, overseeing the creation of plainlanguage summaries of scientific papers and coordinating various initiatives with other CFS KE practitioners across the country. Prior to the pandemic, CFS KE practitioners conducted their work in both digital and in-person formats, where digital formats could include webinars, videos, and newsletters, and in-person work could involve field visits to demonstrate technology, conferences, and collaborating with knowledge users to co-develop project objectives.

Traditionally, the CFS relies on in-person, face-to-face interactions to build trust with knowledge users, such as industry representatives or provincial decision-makers, to promote the uptake of new knowledge and practices for forest management. The pandemic abruptly ended in-person interactions and forced them into digital forms and spaces. It is currently unknown what elements of KE can be preserved or even enhanced by the forced transition to digital interaction. It is also unknown how long the pandemic will render meeting in-person impossible, and whether or not we can expect a return to KE practices in the pre-pandemic way. For these reasons, CFS KE practitioners represent an ideal case study to begin to explore the risks, promises, and pitfalls of digital knowledge exchange in a post-COVID-19 pandemic world.

We use responses from interviews with nine CFS KE practitioners to provide examples. The interviews were faceto-face over videoconference using Microsoft Teams and occurred during the pandemic while respondents worked remotely at home. We draw on 15 semi-structured interviews in our discussion below. Of these 15 , nine interviews occurred between July and November 2020 and were conducted by CB. We did six follow-up interviews (conducted by $\mathrm{JH}$ and TK) with the same group of interviewees in February 2021. These were part of a broader study funded by a Partnership Engage Grant from the Canadian Social Science and Humanities Research Council (SSHRC) to investigate the role of KE in overcoming science-policy gaps within the CFS. Participants were originally identified by the CFS head office. We also used a snowball sampling approach, whereby the original participants that were identified by the CFS Head Office were invited to suggest other relevant people involved in KE in the CFS. This project was approved by the Carleton University Ethics Board (Clearance ID \#112865).

In the interviews, the CFS KE practitioners discussed their work on $\mathrm{KE}$ and their perspective on the lasting impacts of COVID-19. Of the 35 questions asked, we focus on the following questions for the purpose of this article:

(a) Has the COVID-19 pandemic affected KE? (this question was a follow-up prompt to asking about perspective on work/dialogue around KE in the near future); 
(b) What do you anticipate will be the lasting impacts from COVID-19 on KE work? (this question was a prompt about new challenges or benefits of this new norm);

\section{(c) What do you foresee for KE post-COVID?}

We used QSR NVivo 12 for analysis. Data analysis involved coding the interview transcripts inductively and deductively using open and structured approaches (Saldaña 2016). A codebook was created for the entire interview using inductive coding on three interviews that were most elaborate in the responses. For inductive coding, the analysis team (VN, MF, JH \& TK) used both structural and descriptive coding to assign labels to data, which provided an inventory (i.e. codebook) of topics that emerged (e.g. following Blythe and Cvitanovic 2020, p. 4). For this article, we labelled any mention of COVID-19 and KE work as "COVID KE" for the first coding cycle. The second cycle of coding was conducted by VN using inductive coding. Four broad themes emerged related to participant discussions of KE and the pandemic risks and challenges (reported in Sect. 3); loss or importance of in-person, face-to-face KE (reported in Sect. 3); uncertainties (quotes drawn upon for Sect. 5); and opportunities (examples drawn upon for Sect. 4).

\section{What we heard: perspectives about digital KE from the CFS KE practitioners}

In this section, we present and discuss findings from the interviews with the CFS KE practitioners. Challenges and risks identified by KE professionals include loss of interpersonal connections, loss of social learning, being "out of the know", risk of decreased relevance of KE work, and an overall perceived decreased time for KE work individually and for CFS as an organization.

\subsection{Risks and challenges to interpersonal activities and relationships}

Analysis of the interviews found that CFS KE practitioners considered that COVID-19 restrictions on travel and stayat-home orders have interrupted the traditional in-person practices for establishing and maintaining effective KE. Prepandemic, KE often involved gathering at a physical location for an event: conferences, exhibits, site visits, forums, field research, and pubs and restaurants to name a few. Practices of social learning, trust-building, and informal communication have been interrupted with uncertain long-term effects.

The importance of in-person interactions for building relationships, strengthening communication with users, and the integral role it plays in KE was emphasized by many CFS KE practitioners, along with how KE is, and will be affected by COVID-19. As one participant suggested, “...there still comes a time in KE... relationship building moments... where you just can't do it over the screen or online. It's much more of an in-person thing. Human interaction seems to develop a good relationship with people to do this kind of work". (Participant 3).

Participants highlighted how part of understanding or getting to know a topic and exchanging knowledge is to get to know people who have expertise or experiences that are relevant, and to also understand what knowledge is relevant. Not only are in-person meetings more conducive to informal chatter, sharing stories and getting to know someone, but they enable subtle affective exchanges and perceptions. Communication and knowledge co-production involve being attuned to attitudes, to emotional responses to issues of concern, and body signals. It is much more difficult to "read" and interpret the atmosphere in a group in an online meeting, and it is more challenging to respond to subtle social cues.

This sentiment was reiterated by another CFS KE practitioner: The importance of face-to-face meetings are becoming more and more important. The ability to share that research and KE, but [whether] it's applicable to an enduser is what we're losing [due to COVID-19 restrictions]. When you're standing in the woods or riding on a bus with a group, you'll hear a different answer, people will tell you issues that they're having. Nobody wants to tell you right now that they're having a problem regenerating spruce in a clear-cut. The company value is everything, 'it's going great'. But when you're with them walking around looking at stuff, you hear things, and you evaluate, and you find out what the real issues are on the ground. (Participant 5).

Participants also emphasized that some knowledge is experiential and cannot be easily communicated online or remotely: it must be experienced to be understood. Experiential knowledge is often best acquired through in-person workshops and field visits, for example, and one risk is to focus only on knowledge that can be exchanged on online platforms rather than the knowledge that can be exchanged and learned in-person. Further, there is a potential for greater risks for misunderstanding, missed opportunities for relationship building, and risks to precarious or new relationships that require in-depth dialogue and nurturing.

One CFS KE practitioner summarized this issue: I think it's important to listen carefully to people. To try and fully understand 'their problem'. So, if I or anybody truly understands the problem you have to see if your work is truly of any relevance to their problem. Or to have a conversation, a meeting of the minds to see what I know, what we have... and how it fits into the needs of the whatever.... And again, these are things that don't always come about by a single email interaction or a question that you get over a [Microsoft] Teams call. Often, you've got to build a relationship to understand somebody's point of view or perspective or 
needs. That's one thing that I think is an important part of knowledge exchange. (Participant 2).

\subsection{Challenges of going digital: personal disconnection and loss of social learning}

The analysis of interviews also led to the identification of personal disconnection and loss of social learning as other challenges that CFS KE practitioners have faced in relation to the digital transformation of KE. At least part of the CFS's KE work includes three abilities: to work with industry, to demonstrate tools or practices, and to provide support and social learning through in-person site visits or workshops. It seems that for the CFS, the relationships built through these activities ensure the relevance and applicability of CFS research. Exchanging knowledge digitally, which often involves meetings through Zoom or Microsoft Teams, creates challenges for such activities. As one participant described, digital KE creates barriers to having deeper, meaningful conversations: Having those face-to-face conversations... the thing is you can't do that with a video chat. Some people feel more comfortable. You know, I don't have 800 people listening in on my conversation here, I can actually have a one-on-one chat with this individual because I've got an issue with a particular subject. (Participant 6).

Another CFS KE practitioner described their thoughts on an alternative plan to exchange knowledge for an event that had traditionally been in-person: The strategy on that [work with industry on transfer or building capacity with climate models] is-normally a 2-3-day workshop here, bring people here. In the evenings you go for a beer on the river, have a meal, you get to know people, and it just builds trust...This stuff that we're doing, it's not medical research so people need to keep it in perspective, but building the personal relationship is huge. What we're going to do now, we're probably going to do a series of $2 \mathrm{~h}$ seminar, interactive things...It might involve two dozen or so people involved there at Industry $X$. And then we're going to have to figure out how they can do computer exercises remotely.... So that's not gunna [sic] be so good, in my opinion. It'll get done but I don't think it will be a good as it could or should be. (Participant 2).

The general sense from the respondents suggests that they do not believe digital KE can replace in-person interactions and interpersonal connections built with face-to-face time.

\subsection{Risk of not being in the "know": loss of coordination and being up-to-date}

Although the narrative for KE is often about KE with external end-users, CFS KE practitioners suggest that internal KE is just as important. In other fields, this may be referred to as knowledge management, which primarily refers to practices for managing intellectual capital and information flows to achieve organizational objectives (Bennet et al. 2007 pp. 17-19). Working remotely poses challenges for informal and opportunistic conversations among colleagues, resulting in delayed knowledge exchange as well as decreased coordination and efficiency in KE work. Moreover, KE practitioners are also tasked with scoping the horizon, anticipating needs and to some extent creating opportunities to foster more deliberative and reflexive KE. At the CFS, one of the KE practitioners describes how COVID-19 has led to potential decrease in the efficiency in their multi-site, cross-national organization:

Now with COVID, that I tend to be missing out more on - who's who in the zoo. ... I will hear, or read an email that comes out, a notice saying, "we welcome this new person," where usually you'd know something or hear about it beforehand...So that's a challenge for me to make sure that I'm on board or knowing who the new people are, what they're doing, what they're responsible for. (Participant 8)

This sentiment is expanded on by another participant: Yeah, I think with most things we all get pretty busy with our day job and we don't always stop to talk to each other to make sure that we're doing things in a somewhat coordinated way. So, if one group is talking to the National Parks then it's good to communicate that because we might be having the same conversation...COVID obviously being a lot harder because you can't walk down the hall and run into people. (Participant 3).

Without the social interactions and opportunistic dialogue, the KE practitioners risk not "being in the know". Further, challenges may arise in coordinating KE work across regions, as well as leveraging networks to support $\mathrm{KE}$ and needs of end-users.

\subsection{Out of mind, out of sight: risk of decreased time for KE}

The results of our interviews with CFS KE practitioners also suggest there is decreased time and prioritizing for KE if $\mathrm{KE}$ is not "part of the job" or formally recognized. With stay-at-home orders, there are competing demands and indirect impacts on individuals. Some examples are caring for children at home, impacts of delayed services or support, or increased culture of back-to-back meetings (since there is no need to travel to physical locations). Such demands can lead to burnout, decreased mental capacity, and priority shifts. With competing demands, knowledge exchange risks falling down the priority list, as one participant described:

I always have to try and keep the conversation at the forefront because as soon as you - and COVID has 
hurt us a bit in that area - if you're not constantly in front of the researchers and managers with knowledge exchange it starts to wane. (Participant 8)

Further, for some organizations, there may be a risk of viewing the digital acceleration as a solution to many problems including equity, diversity and inclusion, as well as an opportunity to reallocate budget from travels to other areas viewed as higher priority. One respondent shares their sentiment on this: "So, it [COVID-19 impact] adds challenges to doing this stuff [KE] effectively because I think there's a view among some that 'oh you can just do it via the internet'. I don't think you can, but anyway". (Participant 2).

Overall, there is a risk for $\mathrm{KE}$ to maintain priority and effectiveness, if there is a misinterpretation of the value and importance of KE within an organization as well as individual levels (among knowledge producers and users).

\section{Bright spots and opportunities for digital knowledge exchange}

With forced adoption of a digital-first culture, there is an expansion in digital skills and acceptance among individuals, groups, and organizations (Barnes 2020), opening new possibilities and alternative ways to connect as well as enhancing existing virtual gatherings. For example, the CFS consists of research centres across the country and used e-lectures and virtual meetings across the country prepandemic. The increased social awareness of e-conferencing technologies and tools may have a positive impact on the attendance of these existing events. Without the expectation that engagement must come from in-person actions, $\mathrm{KE}$ practitioners have become more creative in how they engage with their communities of practice (McKinley et al. 2021, pp. 5-6). These online interactions may become more frequent, as the desire and expectation for required in-person communication decreases. One of the interviewed CFS KE practitioners offers their perspective on how the CFS has adapted and adopted digital platforms, which can be seen as an opportunity for other organizations to change:

I think we'll have to have new tools or new ways to produce or to do e-lecture or virtual days. We'll have to think about how can we change things so that we will be sure that people are listening, connecting, and not saturating all that stuff. We produce paper and we still print...before COVID, when we were going to congress, people took all the brochures. Now, the younger people don't like that stuff. So, we want to change and produce stuff that people can just pick on their phone and they can have it. (Participant 9)
Behaviour change often happens or can be triggered by significant life event or crisis (Kwasnicka et al. 2016). The pandemic and the forced digital acceleration may thus be an opportunity for individuals, groups and organizations to adapt and innovate new ways to exchange knowledge and meet desired outcomes. Although only one interviewee refers to the influence of generational differences and issues on KE, it is a topic that deserves more in-depth study to explore how digital KE may help or hinder organizations in adapting to generational change.

In Sect. 3, we presented the insights that emerged via the analysis of interviews with CFS KE practitioners. In this section, we primarily draw on our experiences and perspective to discuss bright spots and opportunities for $\mathrm{KE}$ in a digital-first world beyond the CFS. We supplement ideas with examples from the CFS or quotes from the CFS KE practitioners to help illustrate ideas and discussion of potential opportunities for digital KE.

\subsection{Being grounded is not so bad: benefits of no travel}

In-person, face-to-face interactions are deemed to be one of the most effective means of building trust (e.g. Cvitanovic et al. 2021); however, they are also costly. The significant financial costs associated with $\mathrm{KE}$ and all forms of participatory research are well-recognized (Cvitanovic et al. 2019). For instance, a commonly identified challenge to implement $\mathrm{KE}$ in pre-COVID times at the CFS was the lack of travel budgets to engage participatory research as well as building and maintaining relationships. In addition, geographic isolation makes in-person interactions challenging (sometimes unachievable due to access to regions), and sometimes, face-to-face engagements can be interpreted as undermining transparency unless records are maintained for others to access (Cvitanovic et al. 2019, p. 23). The physical demands and costs of travel can exacerbate inequities present in society (Niner and Wasserman 2021), resulting in the exclusion of marginalized groups from KE or other work. Without the requirement of expensive cross-country travel, budgets and efforts can be redeployed to areas where they could be more impactful (including promoting community-led research) or to invest into diversifying approaches for KE.

Last, being grounded and unable to travel has been positive for the environment. The decrease in carbon emissions from less air travel (a considerable requirement in a country as large as Canada) is a major benefit to the CFS and its researchers (Achten et al. 2013; Fraser et al. 2017; Gossling and Humpe 2020). In fact, many scientists think that scientific meetings should continue to be virtual, or have a virtual component, after the pandemic ends as it has provide accessibility for attending from anywhere in the world offering greater reach (Remmel 2021, p. 185). The potential shift 
in perspectives, attitudes, and behaviours towards alternative means to travel may result in opportunities to strategically redistribute travel resources, rethink alternative ways to connect with geographically isolated communities and marginalized groups, and maintain positive impacts on our carbon footprint. There is, however, no one-size-fits-all to approach KE in a digital-first world, and we must continue to be mindful of the value of interpersonal relationships and interactions, particularly for those at early career stages where networking and building collaborative relationships are important.

\subsection{Digital equity: opportunities for access and broader reach}

National and international travel can be costly (Arend and Bruijns 2019, p. 158), time-consuming, and inaccessible (Favaro et al. 2016, p. 3). Travel is particularly inaccessible for those researchers located in remote and low- or middleincome areas who rely on such conferences for KE and networking opportunities. Not only are the cost and travel times reduced by moving conferences online, but the event itself becomes more accessible to diverse researchers who may otherwise be tacitly excluded from engaging in the traditional conference cultures (Hinsley et al. 2017; Niner and Wasserman 2021). A virtual format reduces the barriers to entry for new researchers to engage with knowledge, in spaces where they are safe. In this way, the audience available for KE activities expands beyond the cultural boundaries imposed by forced travel. Further, by removing the spatial and temporal barriers for engagement, KE practitioners can engage with communities from different locations and time zones at once. This also decreases the pressure and cost on communities to formally host researchers and allows them to interact on their own terms, setting their own boundaries, and possibly making consultations more equitable.

\subsection{Digital innovation and knowledge exchange}

The rapid response to the COVID-19 pandemic relied on new and innovative digital solutions across diverse sectors. Many of these tools addressed KE problems identified by CFS respondents- the need for personal connection, maintaining knowledge and communication flows, and stimulating engaging discussions. Here, we survey innovations from areas such as teaching and health care, to highlight digital innovations and tools that have potential to offer new ways of collaborating and exchanging knowledge virtually.

Online learning and communication platforms have grown in recent years, but the COVID-19 pandemic forced educational and health care providers to adapt in-person learning and communication to a virtual space (Golinelli et al. 2020, p. 15; Vargo et al. 2021, p. 15). In the field of education, virtual teaching platforms with interactive features (e.g. live chat, quizzes, file sharing, and breakout discussion groups) provided instructors with real-time feedback about whether the knowledge being communicated is relevant and understood. These interactive features are important for digital KE, particularly for interpreting social nuances and feedback.

Another area of virtual education that has been gaining attention during the pandemic is asynchronous communication (Daniel 2020, p. 93). Digital tools supporting asynchronous communication allow users to record and share video responses to an ongoing discussion. This can confer some advantages over live, synchronous engagement, such as flexibility around differing schedules, fewer technological disruptions, and allows for deeper discussion over longer periods of time (Daniel 2020, p. 93; Su et al. 2020, p. e267). Thus, supporting asynchronous KE opens opportunities to reach broader audiences, accommodate varying needs of users, and be more inclusive.

There are a number of digital options for collaborative KE that have grown in popularity during the pandemic. Some provide a shared space for brainstorming, such as Microsoft Whiteboard, Miro, or Mural, incorporating visual communication tools such as flowcharts, sticky notes, mapping and drawings (Rath et al. 2021, p. 2). Others are expanding the storytelling capabilities of knowledge holders. ArcGIS StoryMaps, for example, can connect advanced GIS mapping tools with pictures, video, and narrative text to communicate about social and environmental projects (c.f. Puri 2020). This introduces the potential for new diplomatic ways to collaboratively share Indigenous and Western knowledge in ways that connect people, knowledge, histories, and place. Through additional technological development and refinement, it has also been suggested that virtual reality may present another way forward for supporting $\mathrm{KE}$ in a digital world, for example, via academic conferences done using virtual reality (Mulders and Zender 2021).

\subsection{Diversification of approaches to knowledge exchange}

The increased culture of a digital world, including adoption, access, skills and innovation in digital and virtual approaches, creates a diversification of approaches to knowledge exchange. For instance, the increased comfort and norm of meeting virtually allows for more frequent meetings at times when expected in-person face-to-face engagements were not possible. In addition to increasing number of meetings, there are opportunities for more rapid and effective response to disturbance events or crises by convening online meetings of people from different places rapidly. This does not replace the need for in-person interactions and events, 
but provides diversified opportunities to learn and exchange knowledge.

Oftentimes, projects operate on a budget where travel to communities, site visits, on-the-ground work and relationship building are limited to yearly visits or less due to high travel costs (particularly in remote areas). In particular, building and maintaining long-term relationships with communities (both Indigenous and non-Indigenous) are integral to successful knowledge exchange and project outcomes. With increased access, skill, and culture of meeting and exchanging knowledge digitally, there are opportunities for more frequent interactions without travelling. Supplementing in-person visits with more frequent digital interactions may enhance the relationship and promote KE.

\section{Looking into the future: sustainability and effectiveness of digital knowledge exchange}

Among the positive impacts of digital-first KE is that it removes the requirement to physically travel and commit large chunks of time exclusively to KE meetings and processes. If considered in isolation, removing these temporal and spatial barriers seems to make KE participation more accessible to a broader range of people. But we cannot consider the potential benefits in isolation of ongoing challenges, which makes the long-term sustainability of digital KE highly uncertain. Looking to the future, we can assume that digital collaboration platforms will continue to evolve and give more options to users. However, in our view, the long-term efficacy of digital KE likely has less to do with software features than with two interconnected factors: (1) the stubborn persistence of the digital divide, and (2) people's abilities to make and maintain meaningful social connections and trusting relationships in the absence of regular in-person, face-to-face contact.

On the first point, the digital divide is a multi-dimensional problem that includes challenges of access, ease of use, and capacity. Marginalized people are less likely to be regular Internet users, and residents of poorer urban and rural areas are less likely to have in-home broadband (Reddick et al. 2020, p. 1). These problems are exacerbated in rural areas where Internet infrastructure is underdeveloped (Maitland 2018, p. 62). Even in rich countries, reliable high-speed connection to the Internet in remote areas is inconsistent and often very expensive (Townsend et al. 2013, p. 583). Metered Internet connections are common in isolated regions, making videoconferencing more costly than in urban and peri-urban settings (Hameed et al. 2018). Access to quiet indoor spaces for digital meetings can be limited due to crowded housing and remote work locations. Lack of day-to-day familiarity with online tools is also an important digital divide, and may limit the participation of older persons, the less formally educated, and people with low technological literacy (Freeman et al. 2020, p. 1947).

On the second point, a major challenge to digital $\mathrm{KE}$ over the long term is the fact that knowledge sharing and exchange as processes rely so strongly on social connections and trust (Reed et al. 2014). However, not all forms of knowledge can be exchanged online. This point is particularly salient for agencies such as CFS, which seek to build and maintain relationships with potential knowledge users beyond the scientific community and beyond government. The knowledge held in oral, experiential and placebased forms is developed and communicated by taking part in practices, by close observation of forests, or by getting acquainted with forestry practitioners in their own working environment, in industry or elsewhere. It is unknown at this point whether online meetings and collaboration can generate and maintain the same kinds of informal exchanges, friendship development, trust building, and networking as face-to-face interactions (Schwarz et al. 2020).

While the COVID pandemic pushed many teams and networks to meet and collaborate virtually, these generally built on existing relationships forged at least partially in-person, as described by a participant: “... when we present ourselves to stakeholders or partners every year, they remember us and sometimes I think the memory of us is very important. So, it's very different than sending email saying do you have something for me? Do you have something from us?" (Participant 9).

If digital $\mathrm{KE}$ is to persist long-term in a digital-first world, people will need to find a way to replicate the social nuances of trust building and contextual understanding that we are accustomed to doing in the physical presence of others. Can we "get to know someone" enough to build trust and goodwill within a virtual environment where conversations are difficult, cameras are turned on and/or off, interruptions and distractions are frequent, and facial and body language cues are difficult to discern? Existing research is preliminary, suggesting that effective digital collaboration is possible for teams of trained knowledge workers within the same organization (Waizenegger et al. 2020). This is definitively not the case for CFS, whose KE stakeholders are economically, culturally, and epistemically heterogeneous. The limitations of digital KE with heterogeneous groups may therefore become more obvious with time if policymakers and practitioners try to implement it as a substitute for in-person relationship building rather than as a complement to unmediated social interactions.

To couch our perspective on long-term sustainability of digital $\mathrm{KE}$ in the context of the CFS, one of the KE practitioners provide some of their forward looking thoughts regarding digital $\mathrm{KE}$ : 
The reliance on technology. I think that's going to be more emphasized rather than let's have 500 people come to a meeting. We're going to have a national meeting online. I think there's going to be less reliance on travel for big meetings. Smaller meetings, perhaps. Travel to a certain degree but I don't see it... that's a real big variable. Because there is a certain amount of travel that's going to be a necessity. I strongly believe face-to-face conversations must be initiated. This business of relying solely on video chat is not healthy and doesn't foster that understanding of the issues. (Participant 6)

There are uncertainties about the post-pandemic world, particularly with respect to KE and the ways in which we enact participatory research approaches to foster shared learnings and enable evidence-informed decision-making processes. The long-term effects of this "reset" from the pandemic are difficult to predict at this moment, but many scholars argue it is a transformation event and it is unlikely that the "old normal" will fully return (Fenwick et al. 2021). A digital-first world may be inevitable, thus strategic thinking, planning and research is important to inform post-pandemic KE. Social-psychologists suggest that behavioural change happens during crisis (Kwasnicka et al. 2016), and thus, we are at a moment in time that is conducive to shaping how we move forward post-pandemic.

It is important to note that $\mathrm{KE}$ is an iterative and reflexive practice, not a protocol. This is critical because $\mathrm{KE}$ is highly context specific (e.g. depending on the topic of KE, scale of issues, stakeholders involved, etc.), and thus there can be no one-size-fits-all approach to how KE efforts are implemented (e.g. Cvitanovic and Hobday 2018, p. 3). Therefore, while some of the expected outcomes (positive or negative) of KE may be known (and planned for) in advance, there is often a mismatch between the aims, expected outcomes and actual outcomes of KE processes (Karcher et al. 2021), and thus unexpected outcomes (positive or negative) can also occur. Similarly, while some of the pre-conditions of successful KE are to some extent predictable, these can also vary significantly across contexts, representing additional challenges to KE practitioners during the digital transformation. However, when implemented effectively and mindfully, KE is also a practice in which outcomes are co-constructed by those who are involved in the meeting and by the extent to which the issues of concerns of participants can be voiced and addressed. The extent to which an online platform enables the expression of issues of concern, allows participants to respond and collaborate, and facilitates learning and the co-construction of relevant solutions is an open question.

\section{Conclusion-considerations for post-pandemic knowledge exchange}

We conclude our perspective essay with the following three considerations to help guide future KE efforts and initiatives in a digital-first world:

1. Consider the double-edge sword of the digital divide and equity In our article, we discussed both digital divide and digital equity. Digital KE promises greater reach, accessibility, and inclusivity. However, total reliance on digital communications and technologies will not suffice due to the digital divide and the challenge in replicating informal interactions, meaningful discussions, and personal connections. Further, data security such as "zoombombing"- the invasion into a non-password digital meetings - continue to be concerns (Schwarz et al. 2020). Without network support and coverage in remote and less developed regions, a digital-first world is surely going to exacerbate the marginalization of people. At the institutional level, practitioners must not lose sight of the importance of on-the-ground work and personal connections.

2. Continue considering user needs Strategies and considerations must be put in place to ensure that the needs of user groups are still met. It is possible that certain users have become adept to digital communications or even prefer it. There is value in revisiting user needs and preferences to identify the best way forward. This is emphasized by one of our interviewees: Yes, stuff is changing and we saw it and we want to evaluate on this. But we have to take the time to think what we can change to respond to the needs of the people who came to see us. After COVID, we will continue to do [original pre-pandemic work] but we have to change I think. (Participant 9)

3. Leverage the diversification of KE approaches and innovations The acceleration in both digital innovation and of user access, skills and acceptance of digital work offers new tools and approaches for the KE toolbox. With increased access, there will be increased diversity of audiences and end-users. KE practitioners may leverage frequency, rapidity, and innovative approaches of gathering people virtually to better respond to user needs and support practice, policy, and decisions.

We conclude our essay with a quote from a KE practitioner on the implications for KE in a digital-first world:

Knowledge exchange is going to be important for us to get out there [on the ground/in-person]. How that's going to happen, I don't know. At this point it's going to be all up in the air, and I don't think it's going to change within the next year. I've gotten involved on 
the national team to put out Program 4. We're hosting it here next year. We're sort of adverse to actually having it virtual because it's just not effective to engage people over video chat. It might be the new reality but it's definitely not something that people are going to be willingly embracing because there is a lot more accomplished at meetings than just the actual meeting itself. (CFS KE practitioner, Participant 6)

According to a KE practitioner, it is their job to optimize the use of all tools available in order to leverage the best possible impact and outcome in the sector. The pandemic has introduced challenges to KE work but has pushed boundaries for diversification of KE approaches, which will enhance knowledge exchange among diverse audiences and user groups.

Acknowledgements We are thankful for our partnership with the Canadian Forest Service, Natural Resources Canada, funded by the Partnership Engage Grant through the Social Sciences and Humanities Research Council of Canada. We thank all interviewees who offered their time and experiences with us. Further, thank you to Dr. Alana Westwood and Kimberly Klenk for their support on this project.

\section{Declarations}

Conflict of interest On behalf of all authors, the corresponding author states that there is no conflict of interest.

\section{References}

Achten WMJ, Almeida J, Muys B (2013) Carbon footprint of science: more than flying. Ecol Indic 34:352-355. https://doi.org/10. 1016/j.ecolind.2013.05.025

Addison PFE, Rumpff L, Bau SS, Carey JM, Chee YE, Jarrad FC, McBride MF, Burgman MA (2013) Practical solutions for making models indispensable in conservation decision-making. Divers Distrib 19(5-6):490-502. https://doi.org/10.1111/ddi.12054

Arend ME, Bruijns SR (2019) Disparity in conference registration cost for delegates from low- and middle-income backgrounds. Afr J Emerg Med Rev Afr Méd D’urgence 9(3):156-161. https://doi. org/10.1016/j.afjem.2019.01.016

Barnes SJ (2020) Information management research and practice in the post-COVID-19 world. Int J Inf Manag 55:102175-102175. https://doi.org/10.1016/j.ijinfomgt.2020.102175

Beaunoyer E, Dupéré S, Guitton MJ (2020) COVID-19 and digital inequalities: reciprocal impacts and mitigation strategies. Comput Hum Behav 111:106424-106424. https://doi.org/10.1016/j.chb. 2020.106424

Bennet A, Bennet D, Fafard K, Fonda M, Lomond T, Messier L, Vaugeois N (2007) Knowledge mobilization in the social sciences and humanities. MQI Press, Frost

Blythe J, Cvitanovic C (2020) Five organisational features that enable successful interdisciplinary marine research. Front Mar Sci 7:539111

Chambers JM, Wyborn C, Ryan ME, Reid RS, Riechers M, Serban A, Bennett NJ, Cvitanovic C, Fernandez-Gimenez ME, Galvin KA, Goldstein BE, Klenk NL, Tengö M, Brennan R, Cockburn JJ, Hill R, Munera C, Nel JL, Österblom H, Bednarek
AT, Bennett E, Brandeis A, Charli-Joseph L, Chatterton P, Curran K, Dumrongrojwatthana P, Durán AP, Fada SJ, Gerber JD, Green JMH, Guerrero AM, Haller T, Horcea-Milcu A-I, Leimona B, Montana J, Rondeau R, Spierenburg M, Steyaert P, Zaehringer JG, Gruby R, Hutton J, Pickering T (2021) Six modes of co-production for sustainability. Nat Sustain. https:// doi.org/10.1038/s41893-021-00755-x

Cvitanovic C, Hobday AJ (2018) Building optimism at the environmental science-policy-practice interface through the study of bright spots. Nat Commun 9(1):3466-3466. https://doi.org/10.1038/ s41467-018-05977-w

Cvitanovic C, Hobday AJ, van Kerkhoff L, Wilson SK, Dobbs K, Marshall NA (2015) Improving knowledge exchange among scientists and decision-makers to facilitate the adaptive governance of marine resources: a review of knowledge and research needs. Ocean Coast Manag 112:25-35

Cvitanovic C, McDonald J, Hobday AJ (2016) From science to action: principles for undertaking environmental research that enables knowledge exchange and evidence-based decision-making. J Environ Manag 183(3):864-874. https://doi.org/10.1016/j.jenvm an.2016.09.038

Cvitanovic C, Howden M, Colvin R, Norström A, Meadow AM, Addison PF (2019) Maximising the benefits of participatory climate adaptation research by understanding and managing the associated challenges and risks. Environ Sci Policy 94:20-31. https://doi.org/ 10.1016/j.envsci.2018.12.028

Cvitanovic C, Shellock RJ, Mackay M, van Putten EI, Karcher DB, Dickey-Collas M, Ballesteros M (2021) Strategies for building and managing 'trust' to enable knowledge exchange at the interface of environmental science and policy. Environ Sci Policy 123:179189. https://doi.org/10.1016/j.envsci.2021.05.020

Daniel J (2020) Education and the COVID-19 pandemic. Prospects 49(1):91-96. https://doi.org/10.1007/s11125-020-09464-3

Evely A, Fazey I, Reed AE, Stringer L, Reed M (2012) Designing knowledge exchange for resilience: how people view and construct knowledge matters. Sustainable Learning Working Paper Series No. 2. http://sustainable-learning.org/workingpapers/newwo rking-papers

Favaro B, Oester S, Cigliano JA, Cornick LA, Hind EJ, Parsons EC, Woodbury TJ (2016) Your science conference should have a code of conduct. Front Mar Sci. https://doi.org/10.3389/fmars.2016.00103

Fazey I, Evely AC, Reed MS, Stringer LC, Kruijsen J, White PVL, Newsham A, Jin L, Cortazzi M, Phillipson J, Blackstock K, Entwistle N, Sheate W, Armstrong F, Blackmore C, Fazey J, Ingram J, Gregson J, Lowe P, Morton S, Trevitt C (2012) Knowledge exchange: a review and research agenda for environmental management. Environ Conserv 40(1):19-36. https://doi.org/10. 1017/S037689291200029X

Fenwick M, McCahery JA, Vermeulen EPM (2021) Will the world ever be the same after COVID-19? Two lessons from the first global crisis of a digital age. Eur Bus Organ Law Rev 22(1):125-145. https://doi.org/10.1007/s40804-020-00194-9

Fraser H, Soanes K, Jones SA, Jones CS, Malishev M (2017) The value of virtual conferencing for ecology and conservation. Conserv Biol J Soc Conserv Biol 31(3):540-546. https://doi.org/10.1111/ cobi. 12837

Freeman J, Park S, Middleton C (2020) Technological literacy and interrupted internet access. Inf Commun Soc 23(13):1947-1964. https://doi.org/10.1080/1369118X.2019.1623901

Golinelli D, Boetto E, Carullo G, Nuzzolese AG, Landini MP, Fantini MP (2020) Adoption of digital technologies in health care during the COVID-19 pandemic: systematic review of early scientific literature. J Med Internet Res 22(11):e22280-e22280. https://doi.org/10.2196/22280

Gössling S, Humpe A (2020) The global scale, distribution and growth of aviation: implications for climate change. Glob 
Environ Change 65:102194. https://doi.org/10.1016/j.gloen vcha.2020.102194

Hameed A, Mian AN, Qadir J (2018) Low-cost sustainable wireless Internet service for rural areas. Wirel Netw 24(5):1439-1450. https://doi.org/10.1007/s11276-016-1415-8

Hardy Y (2002) The Canadian forest service: new directions for science and technology. For Chron 78:57-59

Hinsley A, Sutherland WJ, Johnston A (2017) Men ask more questions than women at a scientific conference. PLoS ONE 12(10):e0185534-e0185534. https://doi.org/10.1371/journal. pone. 0185534

Karcher DB, Cvitanovic C, Colvin RM, van Putten EI, Reed MS (2021) Is this what success looks like? Mismatches between the aims, claims, and evidence used to demonstrate impact from knowledge exchange processes at the interface of environmental science and policy. Environ Sci Policy 125:202-218

Klenk NL, Hickey GM (2011) Government science in forestry: characteristics and policy utilization. Forest Policy Econ 13(1):37-45. https://doi.org/10.1016/j.forpol.2010.08.005

Kwasnicka D, Dombrowski SU, White M, Sniehotta F (2016) Theoretical explanations for maintenance of behaviour change: a systematic review of behaviour theories. Health Psychol Rev 10(3):277296. https://doi.org/10.1080/17437199.2016.1151372

Maitland CF (2018) Now you see it, now you don't: digital connectivity in marginalized communities. Computer 51(6):62-71

Mckinley E, Crowe PR, Stori F, Ballinger R, Brew TC, Blacklaw-Jones L, Cameron-Smith A, Crowley S, Cocco C, O'Mahony C, Mcnally B, Power P, Foley K (2021) 'Going digital'-lessons for future coastal community engagement and climate change adaptation. Ocean Coast Manag 208:105629. https://doi.org/10.1016/j.oceco aman.2021.105629

Mulders M, Zender R (2021) An academic conference in virtual reality?-Evaluation of a socialVR conference. In: 2021 7th international conference of the immersive learning research network (iLRN). IEEE

Nguyen VM, Young N, Cooke SJ (2017) A roadmap for knowledge exchange and mobilization research in conservation and natural resource management. Conserv Biol 31(4):789-798. https://doi. org/10.1111/cobi.12857

Nguyen VM, Young N, Brownscombe JW, Cooke SJ (2019) Collaboration and engagement produce more actionable science: quantitatively analyzing uptake of fish tracking studies. Ecol Appl. https:// doi.org/10.1002/eap.1943

Nguyen VM, Delle Palme C, Pentz B, Vandergoot CS, Krueger CC, Young N, Cooke SJ (2020) Overcoming barriers to transfer of scientific knowledge: integrating biotelemetry into fisheries management in the Laurentian Great Lakes. Socio Ecol Pract Res 3(1):17-36. https://doi.org/10.1007/s42532-020-00069-w

Niner HJ, Wasserman SN (2021) Better for whom? Leveling the injustices of international conferences by moving online. Front Mar Sci. https://doi.org/10.3389/fmars.2021.638025

Puri P (2020) Genomics in society. UBC Faculty of Forestry. https:// genomics.forestry.ubc.ca/

Rath A, Sidhu P, Wong M, Pannuti C (2021) The impetus to interactive learning: whiteboarding for online dental education in COVID-19. J Dent Educ. https://doi.org/10.1002/jdd.12533

Reddick CG, Enriquez R, Harris RJ, Sharma B (2020) Determinants of broadband access and affordability: an analysis of a community survey on the digital divide. Cities 106:102904. https://doi.org/ 10.1016/j.cities.2020.102904
Reed MS, Stringer LC, Fazey I, Evely AC, Kruijsen JH (2014) Five principles for the practice of knowledge exchange in environmental management. J Environ Manag 146:337-345. https://doi. org/10.1016/j.jenvman.2014.07.021

Remmel A (2021) Scientists want virtual meetings to stay after the COVID pandemic. Nature 591:185-186. https://doi.org/10. 1038/d41586-021-00513-1

Saldaña J (2016) The coding manual for qualitative researchers, 3rd edn. Sage, Thousand Oaks, CA

Schwarz M, Scherrer A, Hohmann C, Heiberg J, Brugger A, NuñezJimenez A (2020) COVID-19 and the academy: it is time for going digital. Energy Res Soc Sci 68:101684. https://doi.org/ 10.1016/j.erss.2020.101684

Su MY, Lilly E, Yu J, Das S (2020) Asynchronous teledermatology in medical education: lessons from the COVID-19 pandemic. J Am Acad Dermatol 83(3):e267-e268. https://doi.org/10.1016/j. jaad.2020.06.033

Townsend L, Sathiaseelan A, Fairhurst G, Wallace C (2013) Enhanced broadband access as a solution to the social and economic problems of the rural digital divide. Local Econ 28(6):580-595. https://doi.org/10.1177/0269094213496974

Vargo D, Zhu L, Benwell B, Yan Z (2021) Digital technology use during COVID-19 pandemic: a rapid review. Hum Behav Emerg Technol 3(1):13-24. https://doi.org/10.1002/hbe2.242

Waizenegger L, McKenna B, Cai W, Bendz T (2020) An affordance perspective of team collaboration and enforced working from home during COVID-19. Eur J Inf Syst 29(4):429-442. https:// doi.org/10.1080/0960085X.2020.1800417

Ward V, Smith S, House A, Hamer S (2012) Exploring knowledge exchange: a useful framework for practice and policy. Soc Sci Med 74(3):297-304. https://doi.org/10.1016/j.socscimed.2011.09.021

Young N, Nguyen VM, Corriveau M, Cooke SJ (2016) Knowledge users' perspectives and advice on how to improve knowledge exchange and mobilization in the case of a co-managed fisher. Environ Sci Policy 66:170-178. https://doi.org/10.1016/j. envsci.2016.09.002

Publisher's Note Springer Nature remains neutral with regard to jurisdictional claims in published maps and institutional affiliations.

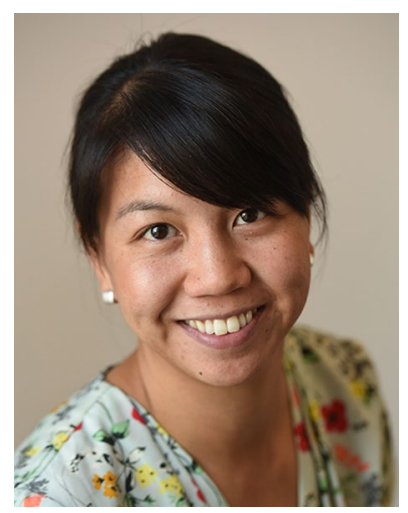

Vivian M. Nguyen is an Assistant Professor at the Institute of Environmental and Interdisciplinary Science and the Department of Biology at Carleton University, located on un-ceded Algonquin Anishinabe territory. Her research focuses on the interface of science, society and policy within the themes of environmental change, natural resource management (including fisheries and forestry), and food insecurity. Nguyen also has expertise in human dimensions of environmental issues and mobilizing ecological knowledge into decisions, policy, and practice. 


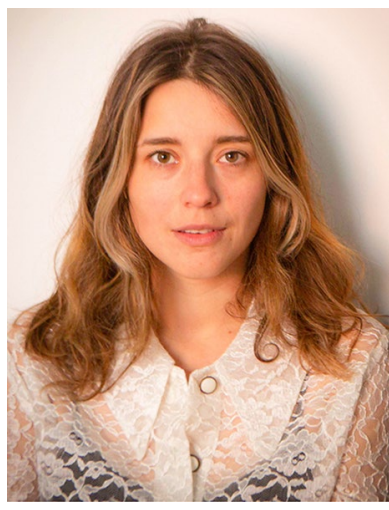

Christina Bell is a Policy Analyst on Canada's Marine Spatial Planning team with the Department of Fisheries and Oceans. She completed her master of science in Environmental Sustainability at the University of Ottawa in May 2020. For her masters research, Christina investigated and documented the practice of knowledge exchange across the country within the Canadian Forest Service. Her interests lie in integrating various kinds of knowledge into natural resource management and environmental policy. Christina is also an active musician and videographer.

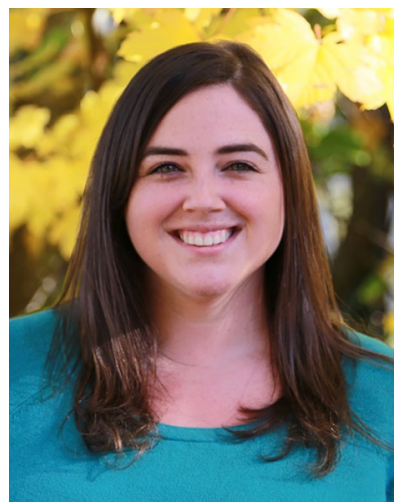

Valerie Berseth is a Research Associate at Carleton University and a doctoral candidate in Sociology at the University of British Columbia. Her research interests focus on social and ecological risks and human-nature interactions in climate adaptation, natural resource management, conservation genomics, and environmental governance.

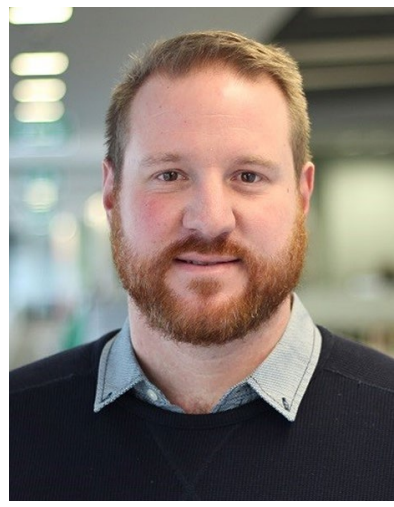

Christopher Cvitanovic is a transdisciplinary marine scientist at the Australian National University, working to improve the relationship between science, policy and practice to enable evidenceinformed decision-making for sustainable ocean futures.

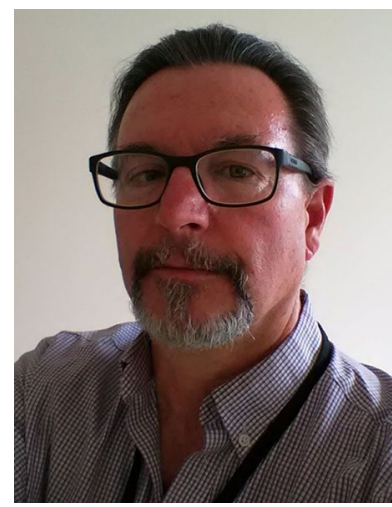

Ray Darwent holds a B.Sc. in Agriculture from the University of Alberta, worked for over 3 and a half years with the Government of Alberta, and for the last 22 years with the Canadian federal public service. In 2012, Ray joined the Canadian Forest Service as Knowledge Exchange Coordinator at the Northern Forestry Centre in Edmonton. Tasked with building a Knowledge Exchange (KE) Program, Ray engaged key forest stakeholders in Alberta and nationally to better understand their research needs, coordinated KE initiatives and activities with CFS researchers and research centres across the country, and delivered CFS research in the hands of forest practitioners in a timely manner. Ray retired from the federal public service in April, 2021.

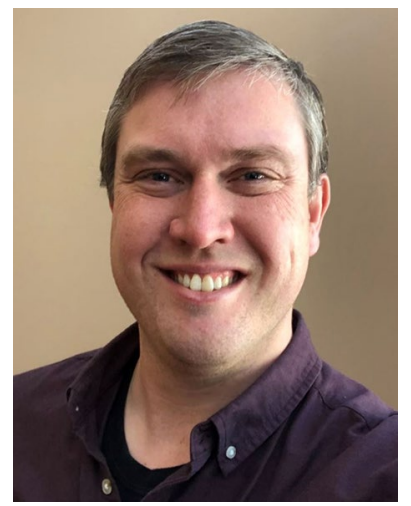

Matthew Falconer is a Knowledge Exchange Analyst in Natural Resources Canada's Canadian Forest Service. He has a Ph.D. in Applied Linguistics and Discourse Studies and is an Adjunct Research Professor in Carleton University's School of Linguistics and Language Studies. His research explores how science is transformed for non-scientific audiences.

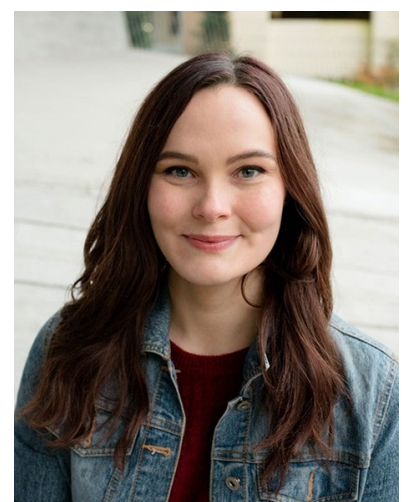

Jenna Hutchen is a PhD student at Carleton University in the Department of Biology. Her research uses an interdisciplinary approach to address social-ecological concerns. In this context, she has studied landscape disturbances such as wildfire and forest insect outbreaks in Canada's conifer forests. 


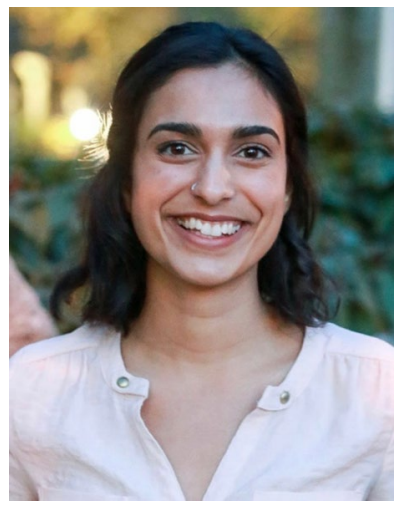

Tyreen Kapoor is a second year M.Sc. student at Carleton University under the supervision of Dr. Vivian Nguyen. Tyreen is studying knowledge exchange practices within Natural Resources Canada to better understand science-policy developments.

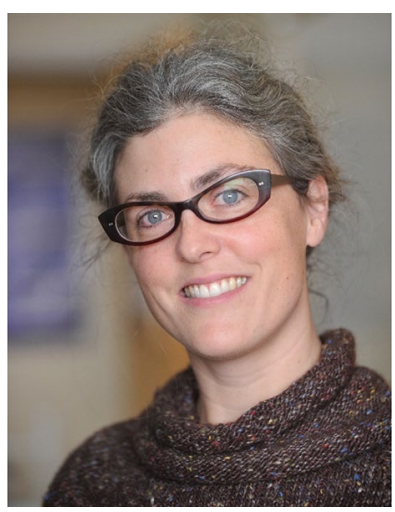

Nicole Klenk is an Associate Professor at the Department of Physical and Environmental Sciences at the University of Toronto. Her recent research has been exploring how storytelling is a way of knowing and making decisions, and how stories as forms of local knowledge may reorient the meshwork that composes environmental research and governance arrangements. Klenk is an academic at an institution located on the territories of the Mississaugas, HuronWendat, and Haudenosaunee.

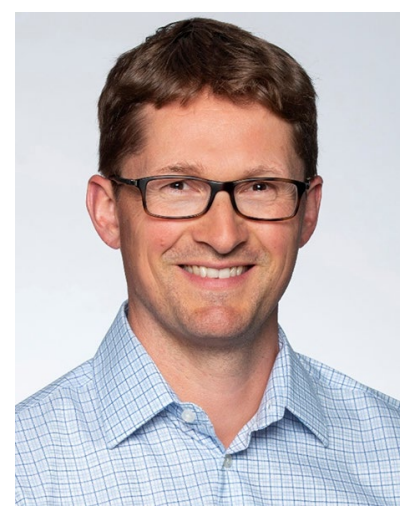

Nathan Young is Professor of Sociology at the University of Ottawa, Canada. His recent research includes studies of climate change politics, conflicts over fisheries and aquaculture, the role of different types of knowledge in environmental management and decision-making, and community resilience in the face of environmental changes. 